\title{
Is there a need for advance care planning in China? - an interview survey of healthcare professionals in the neurology department
}

\author{
Yuanyuan Huang, Hui Liu \\ Institute of Medical Information, Chinese Academy of Medical Sciences \& Peking Union Medical College, Beijing, China \\ Contributions: (I) Conception and design: Both authors; (II) Administrative support: H Liu; (III) Provision of study materials or patients: H Liu; (IV) \\ Collection and assembly of data: Y Huang; (V) Data analysis and interpretation: Both authors; (VI) Manuscript writing: Both authors; (VII) Final \\ approval of manuscript: Both authors. \\ Correspondence to: Hui Liu. Institute of Medical Information, Chinese Academy of Medical Sciences \& Peking Union Medical College, 3 Yabao Road, \\ Chaoyang District, Beijing, China. Email: liuhui@pumc.edu.cn.
}

\begin{abstract}
Backgroundk Family-oriented medical decision-making in China is founded on Confucian familydetermination. The value and necessity of individual autonomy are marginalized in theory and practice. However, the dilemma in end-of-life (EOL) decision-making caused by the lack of advance care planning (ACP) has been increasingly concerned by the public. This study explores the value and necessity of ACP practice in China from the perspective of healthcare professionals (HCPs).

Methods: We conducted semi-structured interviews with five neurological physicians and five neurological nurses from a Grade 3 Class A hospital in Beijing.

Results: The results showed that in situations with a poor prognosis, respondents tended to believe that as part of ACP regarding, a patient's right to refuse invasive treatments should be followed, although all ten interviewees agreed that nasogastric feeding tubes (NGTs) should be maintained. Factors influencing a families' considerations in EOL decision making included prognosis and age, prior similar experiences, others' moral judgement, and the advice of HCPs. Four respondents had experienced patients expressing their wishes for EOL treatments and reported some obstacles in considering these wishes. Seven respondents considered creating an ACP themselves, two respondents doubted it would work in practice, and one respondent thought it was unnecessary.

Conclusions: The differences in the concept and practice of palliative care may be significantly related to the differences in the idea of ACP between Chinese and Western. The family-oriented decision-making model under Confucian culture cannot replace the ACP practice. Clinical situations and therapeutic measures that reveal the necessity and value of ACP should be the priority exploration to advance ACP practice. The practice of ACP and palliative care should be promoted simultaneously at the national level, and legislation for advance directives (ADs) is necessary for mainland China.
\end{abstract}

Keywords: Advance care planning (ACP); Confucian family-determination; end-of-life (EOL); nasogastric feeding tube (NGT)

Submitted Oct 26, 2021. Accepted for publication Nov 18, 2021.

doi: 10.21037/apm-21-3228

View this article at: https://dx.doi.org/10.21037/apm-21-3228

\section{Introduction}

As societies age globally, end-of-life (EOL) medical decisions for geriatric patients pose an increasingly pressing moral issue. Advance directives (ADs) offer patients a legal way to express their wishes on life-saving/prolonging procedures and (or) appointing a proxy decision-maker when they are incapacitated. In the United States, 36.7\% of U.S. adults have completed ADs (1). Advance care planning (ACP) refers to the process that "enables individuals to define goals and preferences for future medical treatment 
and care, to discuss these goals and preferences with family and bealthcare providers, and to record and review these preferences if appropriate" (2). Recording documents are often considered necessary in the process, and ADs are documents carrying legal force. In mainland China, there is no special law for ADs, and in 2006, the first and only web-based ACP process, Choices and Dignity (http://www.xzyzy.com), was launched on the Chinese mainland. Although until early 2021, more than 55,000 people had created ACP documents on this website, this is a low proportion of the eligible population. While the site's founder mentions in an educational video that more than ten cases have been enacted, no formal reports or studies are documenting this to date.

\section{Why might ACP be considered unnecessary in China?}

The family-oriented medical decision-making model in China has a long history and still conforms to the values of most people (3). The four principles of Western bioethics, including autonomy, beneficence, non-malfeasance, and justice, have long been widely discussed in Chinese medical ethics literature, and form the core content of ethical training courses for medical students and healthcare professionals (HCPs). However, the value of patient autonomy is embodied in respecting the wishes of patients and their families. In the view of HCPs and managers, patients and their families are considered one, and their values, together with the professional judgements of physicians, form the basis of medical decisions. When a patient is diagnosed with an incurable or severe disease, the diagnosis and prognosis are often partly withheld from the patient. Doctors relay complete information of disease, optional treatments, and prognosis to family members. Usually, the patient's wishes and preferences are not to be discussed or known, or are known but overlooked. Fan, an East Asian bioethicist, espouses the position of cultural relativism, and he denies the existence of universal ethical principles that transcend specific regional cultures. Fan proposes and demonstrates the value of Confucian family-determination and its broad applicability in East Asian culture. Compared with the western concept of individualism and self-determination, Confucian ethics emphasize the harmonious and dependent relationship between family members. He holds that the family as an independent, ultimate autonomous unit, called Confucian family-determination, is also reflected in the familyoriented medical decision-making model. In this mode, it is the responsibility of the whole family to take care of patients. The moral obligation that family members should fulfill to patients is not determined by the patient's wishes or current needs but by the duty of conscience under the specific relationship between family members and patients (4-8). Fan concludes that families should refuse, for example, to follow $\mathrm{ADs}$ for voluntary cessation of eating and drinking (VSED) (9). On the one hand, compared to the topic of ACP of refusing life-sustaining procedures, the ACP of VSED is an unfamiliar and uncontroversial topic in China, and it is unconvincing to use it as a target to deny the value and necessity of ACP practice. On the other hand, the moral defense of family-oriented decision-making is primarily based on family members' moral obligations or moral positions, rather than the patients' wishes or needs.

The significance of family involvement in medical decision-making is generally emphasized and defended (10). When it comes to the shared decision-making model, the focus is on adjusting the dominance of the doctors and the subordination of the patient; however, both the patient and the family members can represent the patient part (11). Liu et al. constructed and verified the "family-clinician shared decision-making" model in the ICU (12). However, the possible divergences in values, desires between patients and families are generally ignored, even though pieces of literature indicate that patients and their families often have different considerations $(13,14)$. Few legislative, ethical, and practical studies pay attention to and discuss how to ensure patients' wishes be respected and fulfilled in the cases where divergences exist. As a result, the value and necessity of individual autonomy are marginalized in theory and practice.

\section{Why might ACP be considered necessary in China?}

The development of ACP raises the question of whether robust cultural interpretations and justifications partially obscure the broader picture of common practical challenges brought about by modern medical technology whereby death is rendered a choice rather than a natural process. Nearly half a century ago, people in the United States began to reflect on the goal of EOL treatments, and in a landmark event in 1976, the state of California passed the Natural Death Act. Since then, ADs have gradually been protected by special laws in other states, countries, and regions worldwide. In the East, a consensus around withholding/withdrawing life-sustaining procedures is also growing (15). While a 2012 survey revealed cultural 
commonalities among China, Korea, and Japan, in that these countries have a Confucian, family-oriented perspective (16), but only $17.7 \%$ of Chinese respondents wanted to leave EOL decisions entirely to their families (16).

There is a divide on whether the ACP process is necessary between ethical discussion and practical research in China. In ethical literature, the family-oriented decisionmaking model is justified by the assumption that cultural values differ between China and the West; that is, the good and goals pursued by patients' medical decisions are not only for themselves but also for their families. On this basis, an ACP process that emphasizes individual autonomy is unnecessary. However, in the literature concerning medical practices, the value of ACP is predetermined; it focuses on getting people to understand and accept ACP. A literature review in 2019 provides an overview of relevant studies, and ACP advocacy and practices in nursing homes are considered the appropriate environment to begin this process (17). ACP involves a wide range of wishes and preferences, ranging from daily care to life-saving treatments, and the most controversial dilemmas frequently occur in hospitals. This review, and most of the related papers it mentions, focuses on promoting ACP cognition and action but does not focus on the most challenging aspects of ACP practice. The review also introduced a family-centered ACP conversation model widely applicable in Chinese hospitals (17). The problem of this model has been explained earlier. When patients and family members have different opinions, it is predictable that the patients' wishes are likely to be ignored. In addition, this model obscures the significance of encouraging patients to participate in medical decisions through ACP. The taboos on death and the telling of harsh truths are widely emphasized as important regional cultural factors, and death education is considered crucial (17). These aspects are undoubted but perhaps overemphasized in previous studies, as taboos on death are also common to other cultures (18). The values in traditional Chinese culture are multifaceted, and ACP should not be seen as evidence of a one-sided negative attitude towards natural death. Besides, taboos around revealing the truth about their condition to patients stem from avoiding harm. Nevertheless, ACP practice concerns an advanced arrangement of hypothetical future situations, conducive to avoiding the challenge of telling the truth.

In previous studies, questionaries were mainly used to elicit the attitudes of HCPs and the general public towards
ACP. Few in-depth research into situations revolves around actual experiences and perspectives with the ACP practice and the more specific reasons behind these realities and beliefs. Furthermore, compared to the widespread concerned issue on how to get people to know and discuss ACP, another neglected issue is why the wishes of those with arrangements are still ignored. Considering the limitations of existing studies, the novelty of this paper is reflected in that it takes a different, micro perspective on this subject by interviewing HCPs to elicit a specific understanding of ACP. Without pre-assuming the value and necessity of ACP, it interviews the HCPs who may have clinical experience similar to a case that has sparked widespread controversy in recent years. Besides, by calling on the clinical experiences of HCPs, it also provides actual examples of patients expressing their wishes and the role of those wishes in their EOL decisions. As a result, it presents various factors and their associations related to ACP attitudes, opinions and implementation barriers in an objective and specific way.

We present the following article in accordance with the SURGE reporting checklist (available at https://dx.doi. org/10.21037/apm-21-3228).

\section{Methods}

\section{Aims}

This study explored the necessity and value of promoting ACP practice in China, aiming to understand: (I) the views of HCPs on Mr. P's ACP and EOL treatments; (II) the general considerations of families in EOL medical decisionmaking; (III) the experiences and views of HCPs dealing with the expressed wishes of patients; and (IV) the views of HCPs on creating ACPs for themselves.

\section{Design}

We used a semi-structured interview for two reasons. First, we expected the views of HCPs on ACP and EOL medical decisions to be complex rather than definite, rendering quantitative research inappropriate. Second, through semistructured interviews, we could focus on the core questions about experiences, subjective attitudes, and opinions related to ACP and EOL medical decision-making. The semiopen form also allowed respondents to state more facts and details around the relevant issues, which enabled a comprehensive understanding of the relevant factors. 


\section{Identifying respondents}

We identified respondents based on five considerations: (I) the HCPs in the neurology department encounter ethical dilemmas like Mr. P's. They receive patients with strokes and dementia who often experience a loss of mental capacity and encounter EOL medical decision-making problems as the disease progresses. (II) The clinical experiences of HCPs can reflect the general views of patients and their families. (III) As the first recipients of the concept and practice of palliative care and ACP, the views of HCPs can reflect the current understanding and practices of ACP and EOL decision making in China. (IV) Although families are the final decision-makers, the views of HCPs significantly affect the decisions made by family members. (V) Primary healthcare facilities providing hospice and long-term care are in great demand, and there is a significant supply shortage. Hospitals cannot offer long-term hospitalization, and patients with adequate financial support often move from hospital to hospital or are discharged and readmitted frequently. Chinese primary healthcare facilities or nursing homes have not yet played a powerful role in providing long-term care for disabled, geriatric, and terminally ill patients; hospitals are still the most appropriate locales to learn about the related ethical dilemmas.

\section{A controversial case}

The case of Mr. P's ACP case has aroused ongoing public discussion, reflecting a common dilemma in China, providing an entry point for the interview content.

\section{The first nasogastric feeding tube (NGT) decision}

In 2014, Mr. P, 87-year-old, created an ACP stating that he did not want to be resuscitated, intubated, or admitted to the ICU when critically ill. In 2015, he suffered a stroke that left him with dementia, although with the care of his wife, Mrs. Q, he continued to live a chaotic but happy life. One day, he fell into a coma with a high fever, and as doctors suspected lung infection, they recommended providing NGT. While Mrs. Q believed that Mr. P would not want to receive NGT according to what she knew of him, Mr. P's three children from a previous marriage asked doctors to provide NGT for their father. After recovering from the acute infectious disease, Mr. P continued to live in the care of Mrs. Q, would laugh when he played a game with her, and appeared to be attracted by pictures.

\section{The second NGT decision}

In 2016, Mr. P was barely conscious after suffering a more severe stroke. Doctors told Mrs. Q and Mr. P's three children that while intubation could prolong his life by several years, it was improbable that he would react in a meaningful way manner again. While Mrs. Q insisted on following Mr. P's wish, Mr. P's children disagreed, and eventually, Mrs. Q compromised with their wishes (or, as she said, with society). Mr. P was intubated and never regained consciousness until his death in May 2019, leaving Mrs. Q with tremendous guilt and misery for not having the courage to follow his wish.

In 2018, Mrs. Q wrote a book documenting this process and made her ACP public, calling for a broader discussion on the rights of patients in making EOL decisions (19).

\section{Interview outline}

\section{HCPs' perceptions of the first NGT decision in the} case of Mr. P

Q1: Do you think Mr. P should have received NGT the first time? Why?

HCPs' perceptions of the second NGT decision in the case of Mr. P

Q2: Do you think Mr. P should have received NGT the second time? Why?

Mr. P's children refused to follow their father's ACP to withhold NGT because they were emotionally incapable of letting their father starve to death. It is still controversial whether NGT should be the default treatment (20), and it may be preferable to other invasive procedures.

\section{HCPs' perceptions of other life-saving/sustaining treatments in the case of $\mathrm{Mr} . \mathrm{P}$}

Q3: In the second instance, do you think Mr. P should have received other life-saving/sustaining procedures? Why?

\section{HCPs' experiences of families' considerations in EOL medical decision making}

Q4: According to your clinical experience, what are family considerations in EOL medical decision-making? Could you relay something that impressed you deeply or that you disagreed with? 
Table 1 Respondents' backgrounds

\begin{tabular}{lcccccccccc}
\hline Alias & Ye & Fe & Ji & Ho & Li & Da & Le & Ya & Yi \\
\hline Profession & NP & NP & NP & NP & NP & NN & NN & NN & NN & NN \\
Gender (M/F) & M & M & F & F & F & F & F & F & F \\
Years in job & 9.5 & 19 & 23 & 16.5 & 9 & 4.5 & $10.5^{*}$ & 3.5 & 5 & $8.5^{\#}$ \\
\hline
\end{tabular}

*, Le worked for 5.5 years in the general internal medicine department and 5 years in neurology; *, Yu worked for 4 years in internal medicine and 4.5 years in neurology. NP, neurological physicians; NN, neurological nurses; M, male; F, female.

\section{HCPs' experiences of patients' wishes}

Q5: Have you ever experienced a situation where a patient had an ACP or had expressed their wishes regarding future treatment and care plans?

\section{HCPs' perceptions of ACP}

Q6: Would you consider constructing an ACP for yourself in the future? Why?

\section{Data collection}

A total of ten doctors and nurses from the neurology department of a Grade 3 Class A hospital in Beijing volunteered to participate in this study, with researchers establishing contact and arranging interviews with respondents through WeChat. By undergoing a telephonic or face-to-face semi-structured interview, each participant was interviewed once, and each interview lasted about 60 minutes. The hospital is a general hospital, which does not have any characteristics that are particularly related to the research topic. As the selection of the interviewees was random based on the voluntary principle, the views and attitudes of the interviewees were considered to represent the general views of physicians and nurses in large general hospitals in China. All interviews were audio-taped, informed consent was obtained. After the tenth respondent had completed their interview, a decision was made by researchers not to increase the number of interviewees, as the information obtained to that point had become largely repetitious and was considered broadly representative of views on the subject (Table 1).

\section{Data analysis}

We conducted data analysis according to the following steps: (I) We converted the recording into text using automated transcription software. (II) We proofread the text data according to the recordings. (III) We grouped these data into question-based categories. Q1-Q3 explored the respondents' views and were designed to answer the first research question. There were two comparative dividing lines; Q1 and Q2 compared the impact of disease prognosis on HCPs' views of Mr. P's wish; Q2 and Q3 compared respondents' views on NGT and other life-prolonging treatments, ultimately affecting their views on Mr. P' ACP; Q4 and Q5 were the clinical, experience-based questions designed to answer the second and third research questions, respectively; and Q6 was designed for the fourth question, which explored respondents' views on creating ACP for themselves. (IV) We analyzed the data with qualitative content analysis. (V) By reviewing and discussing the data repeatedly, we reached a consensus on the findings, drafted and revised the results and conclusion accordingly. (VI) We translated, proofread, and quoted some representative context in the paper.

\section{Ethical considerations}

All procedures performed in this study involving human participants were in accordance with the Declaration of Helsinki (as revised in 2013). The study was approved by the ethics committee of the Institute of Medical Information, Chinese Academy of Medical Sciences \& Peking Union Medical College (the number of the approval: IMICAMS/02/20/HREC) and informed consent was taken from all the participants. The article's corresponding author, Hui Liu, approached potential participants. All potential interviewees were informed regarding the research objectives, processes and plans, anonymity in the study, the time and methods of recording, storage, and destruction of the interview materials, and the right of participants to withdraw from the interview at any time. To protect the privacy of respondents, they were referred to by twoletter aliases (they were not the respondents' initials). No respondent received financial or any other incentives for participating in the survey. 


\section{Results}

\section{HCPs' perceptions of the first NGT decision in the case of} Mr. $P$

All respondents would have chosen NGT for Mr. P were they his family, considering his condition was a treatable, reversible condition with a good prognosis. As "prognosis" was frequently mentioned, a further question emerges: "What is a good prognosis?". All respondents agreed that the "bottom line" was maintaining consciousness with no great suffering. Retaining the ability to communicate with others is an ideal prognosis. For example, Yu said, "the main first is his consciousness [...]. The other is overall functions like be can communicate with you [...]. The more function she maintains on a conscious basis, the better." Ye said that the premise of quality of life (QoL) is consciousness. Moreover, if the patient can communicate with his family, it is not too much to rescue him actively. If the patient is irreversibly unconscious, then there is no need to rescue him aggressively. A further question was whether a demented person who often appears to be in a happy state could be considered to have a low QoL. All respondents agreed that dementia does not significantly reduce QoL, and it should not, in any sense, affect medical decisions.

\section{HCPs' perceptions of the second NGT decision in the case of Mr.P}

\section{The provision of the NGT was uncontroversial}

All respondents agreed that Mr. P should have received NGT with a poor prognosis because they believe patients cannot starve to death, and NGT is a general and simple procedure that would not cause undue pain or burden, which is quite different from endotracheal intubation and CPR. For example, Le said that NGT provides fluids that prevent electrolyte disturbances and reduce the possibility of lung infections. Moreover, Le said, "if [these] relatively non-invasive [procedures] are not used, it feels like [we're] doing nothing for his life." Yu mentioned the side effects of NGT, including that "patients being unable to taste the food", "damage to the mucous membranes of the patients' throat and oesophagus", and "stronger physical restraints are needed to prevent the patient from removing the tube". However, Yu did not think these side effects constituted reason enough to withhold NGT.

A further question is whether NGT should be provided for people with advanced dementia with dysphagia. All respondents believed that NGT should be provided to avoid the increased risk of pneumonia caused by hand-feeding, and it feels emotionally unacceptable to leave the patients dehydrated and hungry. For example, Ho said that it "is out of respect for life, their life is not over; you cannot say that because someone is demented, then let them starve to death."

\section{Percutaneous endoscopic gastrostomy (PEG) was generally unacceptable}

For the respondents, artificial hydration and nutrition (ANH) mainly referred to NGT. Li mentioned PEG, and she believed it was a better way of nourishing incompetent patients because nurses must often place restraints on their hands to prevent them from removing the NGT, which is cruel. Li also said that the Chinese poorly accept PEG, but it is highly accepted abroad. Yu stated that PEG is invasive and carries unacceptable risks because the stomach needs to be perforated, and food may enter the abdominal cavity through the gastric fistula. Doctors rarely recommend PEG, and few families choose it because it is considered invasive.

\section{HCPs' perceptions of other life-saving/sustaining treatments in the case of Mr.P}

\section{Three respondents had an aggressive attitude toward EOL treatments}

$\mathrm{Fe}, \mathrm{Ho}$, and $\mathrm{Li}$ believed that even if the prognosis was poor and the patients had ACP to refuse NGT and other invasive therapies, they should still receive these treatments. Ho said, "I have been a doctor for many years; I have never encountered anyone who took an ACP and came to say to me that they refused to be rescued. It is a completely different culture; people say it is better to have a bad life than a good death." Ho believed that it was challenging to enforce ACP in Chinese culture. Fe's answer was entangled: "No one wants to receive these measures, no one wants to suffer [...] all people want to die peacefully." However, Fe said that this was just an estimate of their current condition if the prognosis is bad. Many people were worried about becoming vegetative, but a miracle may happen. No one is a prophet, and $\mathrm{Fe}$ would rescue actively and give every life an increased chance of survival. Li said, "If the prognosis is not very good [...], if I were the family, I think I might still be active in the treatments [...]. If the ACP was not followed the first time, I do not think it would mean much the second time. It is not a new decision made after the first intubation. You do not know the patient's current wish." Li also said that if a patient is in a severe coma with a minimal chance of waking up, it is not appropriate for doctors to withhold treatment unless there are objective indicators and 
evaluations that the brain has been irreversibly damaged, and the patient is genuinely unconscious and cannot wake up again. For $\mathrm{Fe}$ and $\mathrm{Li}$, the patient's chance of reversal, rather than continuing a painful, unconscious life, was a more significant point of consideration.

\section{Seven respondents held a sceptical attitude toward EOL treatments}

Seven respondents said they would hesitantly agree to withhold other invasive procedures when the prognosis was poor, if they were Mr. P's family. For example, Yu stated that as a family member or a doctor, the NGT is acceptable, and procedures such as ventilators and CPR are unnecessary in the terminal stage because they only add to the patients' suffering. Le said, "The main [problem] is, maybe I think that the Chinese idea is to save their life as long as they have a breath [...]. If [the treatment] is invasive, it feels like [too much]. Mr. P did not want to suffer [...]. Maybe it is more acceptable to create an ACP like this." It is worth noting that the role of the respondents affected their views on EOL treatments. For example, Yu said, "I think for myself, the quality of my life is not high, I am alive. Yes, but it also will drag others to increase the burden [...]. I would rather not choose to live in such pain [...]. However, if as a family member [...] will not give up [...] at least be is still here."

\section{HCPs' experiences of families' considerations in EOL medical decision making}

In the absence of legally enforceable $\mathrm{AD}$, the family's decision is decisive. From the perspective of HCPs, it also helps to understand the family's considerations and preferences.

\section{Prognosis and age}

Most respondents agreed that it was difficult for families to decide whether invasive procedures should be performed when the prognosis was poor. For critically ill patients, their families decided on their treatment plans. If the families decided to refuse an aggressive plan, when the time came, the HCPs would only provide some non-invasive treatments to alleviate the patient's suffering after reconfirming consent from the families. For example, Le said that usually, for those who refuse invasive resuscitation, a catheter and NGT are still attached to the patients, and medication to maintain blood pressure and heart rate is still administered, but if the patients suddenly become breathless, a ventilator is withheld. Yi said that when the patient can only rely on a ventilator to live, the families generally do not consider aggressive resuscitation measures. Besides, families were more likely to forgo aggressive treatments for older patients. For example, Yu said, "for young patients, it is generally very active to provide them treatment [...]. Family members may find it more difficult to accept a young person leaving. If an older person is in his 80s or 90s, he may have more underlying diseases. We may make a relatively simple decision, and we may prefer the kind of palliative therapy. However, as a young person [...], I think most people would not accept [the palliative care]." Ye said that there were more possibilities of reversal for younger patients who have good physical fitness, and their families would like to choose aggressive treatments to fight for any chance of survival. Age is a consideration because of its association with prognosis.

\section{Families' similar experiences}

When families are unprepared to become decision-makers on EOL treatments for patients, it is uneasy to recognize when forgoing life-saving/sustaining procedures are preferable, often resulting in extended, tangled, challenging decision-making processes. Most families eventually chose to let their loved ones go after trying aggressive treatments several times or being sure that the patients had suffered dramatically in the process and there was no possibility of reversal.

\section{Other's moral judgement}

When the prognosis was poor, Ji said that some family members, especially adult children, may need to avoid negative moral judgments such as unfilial and show a positive (aggressive) attitude towards their relatives and friends.

\section{HCPs' views and suggestions}

There were two unreasonable situations for HCPs. One was families refusing NGT or other invasive treatments when the prognosis is good, and the other was demanding aggressive therapies when the prognosis is poor. Generally, doctors convey their suggestions by emphasizing a good or poor prognosis, influencing families' decisions.

\section{Other factors}

A small percentage of families will refuse treatment when the prognosis is good. This may be because of economic problems or the belief that these medical techniques are burdensome. When the family insists, HCPs will follow the 
families' decisions.

\section{Aggressive cases}

Le mentioned a situation that was impressive to her. The patient was 98 years old with a good pension, and the cost of treatment was not a consideration. Although he was possibly in a vegetative state, his children sent him to different levels of hospitals, depending on his condition and provided him with all kinds of procedures. He had been in the ICU several times and had undergone a tracheotomy. Le said she felt sorry for him, and she doubted whether he ever expressed a wish to live like that.

Contrary to the opinion of most respondents, Li believes that she encounters more families prematurely abandoning active treatments. It may be due to her belief that it is only reasonable to abandon life-sustaining measures when the patient is determined to be completely unable to retain consciousness. However, she mentioned one exceptional case which she felt represented unreasonable persistence in which a brain-dead patient was kept breathing by machine for 3 to 4 months. Da mentioned a case that an older man's family was so aggressive in resuscitating him that he ended up in a coma in ICU for over two years.

\section{HCPs' experiences of patients' wishes}

Four respondents encountered patients who had ACP or expressed their wishes. Fe said some patients told him they refused to be rescued when they had a poor prognosis, although he did not consider their informal directions. Li said that families would consider the desires of patients, but the prognosis was the primary deciding factor. Ye has met some geriatric patients who verbally expressed their wishes, and when they had only one child, their wishes were more likely to be considered. $\mathrm{Li}$ and $\mathrm{Da}$ reported two relative cases.

\section{Improperly following the ACP}

Li reported a case in which a patient with a massive cerebral infarction had expressed to her children that she refused to undergo any invasive procedures. One day later, she developed severe pneumonia. Her breathing was labored, and she needed immediate respiratory support. Considering her wish, her children only accepted a noninvasive ventilator unsuitable for her delirious condition, which caused her to develop severe distension and oedema. Finally, her children agreed to use endotracheal intubation, and she passed the acute phase and regained her sanity without blaming her children for disobeying her wish.

\section{Disobeying the ACP}

Da mentioned a case in which a patient with a cerebral infarction was suddenly in a critical condition. Her daughter asked the doctor to try all rescue procedures, after which his vital signs returned to a stable state. After that, his daughter told $\mathrm{Da}$ that her father had told her that he did not want to be taken to the hospital if his condition suddenly deteriorated. He did not want to be in bed with all kinds of tubes because that would be a qualityless, undignified life. However, when the time comes, she wants her father to be alive, whether conscious or not.

\section{HCPs' perceptions of ACP}

\section{Three respondents did not consider making an ACP for themselves}

Ho said, "I do not think it is necessary to create an ACP, probably for the general person, there is not too much entanglement [...]. For most Chinese people, their desire to live is powerful." Fe believed ACP to be a good idea that was respectful of patients' preferences and helped families make EOL decisions. Still, he thought the medical decisionmaking process was a comprehensive judgment of a specific situation. He worried that simply following a patient's wish would lead to a wrong decision and deprive the patient of survival. In the case discussion, Ji stresses doctor-patient disputes and lawsuits, and she also believes that ACP is necessary. Still, she does not consider creating an ACP because it is challenging for HCPs to enforce it.

\section{Seven respondents expressed a positive attitude toward ACPs}

Seven respondents said they would create an ACP refusing invasive treatments, and the execution conditions included irreversible unconsciousness and the inevitable suffering of great pain. However, they would not refuse non-invasive therapies, including medication, NGT, and urethral tubes. The reasons for creating ACP included avoiding imposing a significant burden on the families (Yu); avoiding all kinds of complications and suffering (Ya); avoiding staying in bed without dignity (Le); reducing families' psychological burden in the decision-making process (Li, Ya, Da); and fulfilling their wish or making decisions about their own life $(\mathrm{Da}, \mathrm{Yi})$. These reasons are very similar to those behind the 
establishment of ACP in other countries (21).

\section{Old age increases the need to create ACP}

All respondents are not currently considering creating ACP and believe they are too young for doing this. Yu said that if she were very old, for example, in her 80 s or 90 s, she would refuse invasive treatments by creating an ACP. Ye said that if the patient were 80 , their ACP would probably have been fulfilled, but if they were 20, there would be a high probability that their family would not follow his wish. Ye believed that a 20-year-old patient in a vegetative state, if well cared for, can live many years, and all sorts of things could turn around with the progress of medical technology in the future.

No respondents quit during or after the interview, and the data of all ten respondents were included. Our interview findings are consistent with the previous studies, which can prove that the views and attitudes of respondents in this study are representative. However, our study mainly focused on patients in the neurology department, and these data may be different from other departments. In this interview, most respondents believe that the QoL of terminally ill patients suffering from severe physical pain is lower than that of patients who lost their rationality or self-care ability. Therefore, in the oncology department, the necessity and value of the ACP may be more prominent, and HCPs and family members may have different attitudes toward ACP. It suggests that the oncology department is an appropriate place to explore the value and practice of ACP.

\section{Discussion}

The necessity and value of ACP depend primarily on the particular clinical situation and treatment involved, which vary significantly across cultures. Therefore, we need to discuss in the current situation in China, when it comes to what conditions and treatments, ACP is acceptable, necessary and valuable. And explore the specific barriers to achieving this value in a particular situation and reinforce these issues accordingly.

\section{When the prognosis worsens, ACP becomes increasingly important}

The responses regarding Mr. P's second admission indicate that QoL is an increasing concern in EOL treatments. The continuation of life is not always a priority, which is a concern often seen in Western countries. When the precedent autonomy of a patient conflicts with their current wish or well-being, prioritizing precedent autonomy is the dominant position adopted in Western ethical discussions (21-24), although this position remains controversial (25). The legislative practice of ADs of most countries and regions strictly limits the execution conditions to several situations, including terminal disease, irreversible comas, permanent vegetative states, and intolerable suffering caused by an incurable illness. Since the age of the Hippocratic Oath, doctors have been trained to place the interests of their patients first. However, with this aim in mind, doctors are often unsure of the right choice in these situations and respecting the autonomy of patients becomes an overriding value.

\section{HCP's perception of palliative care, especially NGT, is lagging, influencing their judgements and families' decisions}

Compared to other EOL procedures, the United States has undergone a more extended transition toward ANH because it is particularly emotionally sensitive (26). A 2003 survey shows that $34 \%$ of nursing home residents with advanced dementia in the US were tube-fed without sufficient evidence to prove that an ANH could prolong life or improve QoL $(27,28)$. Palecek et al. propose renaming the process of withholding or withdrawing ANH "comfort feeding only" to eliminate the moral discomfort evoked by the wording "do not feed" or "do not try everything possible" (29). The percentage of nursing homes in American housing residents with severe dementia that used feeding tubes in 1 year dropped to $5.7 \%$ in 2014 (30).

A study suggests that HCPs in China "might be unfamiliar with palliative care options or reluctant to broach palliative care topics." (31). The gap between the views of respondents and the international consensus on NGT was striking. Compared with long-term NGT, PEG has long been reported to provide better nutrition with fewer complications (32-34), requires less cooperation from patients, and is more suitable for patients with dementia $(35,36)$. However, in Asia, NGT is a more conventional and recognized form of long-term enteral nutritional support $(32,37)$. The understanding of palliative care by HCPs is inadequate, indicating a significant room in China to promote ACP and change public views on EOL treatments. Since 2017, China has begun to push the development of palliative care by issuing policy documents and developing pilot palliative care programs. HCPs will provide a more 
objective understanding of EOL treatments. It is reasonable to expect that more people will choose to create ACP, and it will be more valuable and useful in more contexts. However, there has been a lack of action, study, and policy in China to advance the ACP practice. The close relationship between palliative care and ACP should be recognized and both should be promoted simultaneously at the national level.

\section{Confucian family-determination cannot explain the view that ACP is unnecessary}

Compared to other East Asian countries, there is greater acceptance of EOL treatments in China (16). It is also one reason why Mainland China ranks $72^{\text {nd }}$ in the 2015 Quality of Death Index among 80 countries and regions (38). Providing patients with aggressive life-saving/sustaining treatments in China often means trying everything, marking filial piety and good conscience. However, this connection or judgment does not come from culture itself but from the fact that the concept and practice of palliative care in China lag behind other societies. The moral requirement of filial piety is for families to make an informed choice that is good for their parents, not a misperceived choice. ACP process enables patients to identify their goals and values on EOL treatments and helps families prepare as future agents for the patient by assisting them in understanding EOL medical decisions better. As both families and patients often undergo extended and painful processes before a patient dies, the ACP process would be helpful for both patients and their families. Some respondents worried about the practical challenges of ACP, and web technology has great promise for helping users create, revise, and share their ACPs and encourage families and HCPs to participate in the process (39). China should promote web-based, simple, humanized information on ACP suitable for different groups.

\section{Legally binding $A D$ is necessary}

Western countries have found that $\mathrm{AD}$ has many drawbacks. For example, witnesses or notaries are required, which increases the inconvenience of modifying the $\mathrm{AD}$, and its fixed legal text lacks clinical practicality. ACP and its informal documents are better for families and doctors to understand and translate the patient's goals and values into specific clinical situations (40). In East Asia, the wishes, preferences, and decisions of families are fully respected, and those of patients are often ignored by families and
HCPs. While, in many cases, doctors agree with the wishes of patients, they have no legal basis for denying the different, unreasonable decisions made by families. Korea implemented the Life-Sustaining Treatment DecisionMaking Act in 2018, which increased the rate of patients' self-determination (41). Making ADs legally binding might be necessary, and a significant challenge is how to limit the unreasonable intervention of families while fully respecting their participation. In 2019, Taiwan implemented the Patient Rights to Autonomy Act (this law was announced on January 6, 2016, and carried out on January 6, 2019; see https://www.ly.gov.tw/), requires that at least one family member be involved in the discussion when the $\mathrm{AD}$ is made, which considers the Confucian values of family. It also stipulates that families should not interfere with doctors' compliance with the patients' wishes or ADs. Doctors or hospitals have no legal liability when they comply with the Act, and relevant provisions encourage and guarantee HCPs to defend a patient's right to a good death. Mainland China should also advance the legislative process of ADs by emulating legal practices in other regions of Asia.

In this study, the influences of law, HCP's views on EOL treatments, family's participation, culture and other factors in EOL medical decision making are specifically presented, and the necessity and value of ACP in this process are objectively elaborated. In particular, this study found that the differences in the concept and practice of palliative care may be significantly related to the difference in the idea of ACP between Chinese and Western. Finally, the corresponding suggestions are put forward given these present situations and challenges.

\section{Limitations}

This study was conducted in a Grade 3 Class A hospital in Beijing. Respondents can reasonably be assumed to have had an aggressive attitude toward prolonging patients' lives and seeking any chance of survival, which was more pronounced among the doctors interviewed, such as Ho and Fe. The study's limitations indicate a need for further research with HCPs at primary care facilities or nursing homes responsible for the long-term care of patients. Pilot facilities were launched in some cities in mainland China in 2017, which can be used as interview sites for the next step in research on ACP. Additionally, it would be helpful to seek long-term follow-ups and case studies on patients that seek to understand the views of the patients and their families. According to our respondents' descriptions, economic 
reasons may have influenced decision-making, although insignificant. Our results and discussions may exclude the influence of economic factors and the patients and families who had no options due to financial constraints because they may not be the kind of patients that the respondents regularly deal with who could receive more advanced treatments. They may constitute most of the population in China.

\section{Conclusions}

In China, family-oriented medical decision-making is founded on Confucian family-determination. There has been a lack of action, study, and policy to advance ACP practice. The value and necessity of individual autonomy are marginalized in theory and practice. From a micro perspective, this study found that while HCPs highly accept ACP when the prognosis worsens, their perception of palliative care, especially NGT, is lagging, influencing their judgements and the decisions made by families. Confucian family-determination cannot explain that ACP is unnecessary and that the family-oriented decision-making model cannot replace the ACP practice. Clinical situations and therapeutic measures that reveal the necessity and value of ACP should be the priority exploration to advance ACP practice. The practice of ACP and palliative care should be promoted simultaneously at the national level, and legislation for $\mathrm{AD}$ that balances family participation with patient autonomy is an urgent necessity for mainland China.

\section{Acknowledgments}

We thank the ten physicians and nurses who participated in this study.

Funding: None.

\section{Footnote}

Reporting Checklist: The authors have completed the SURGE reporting checklist. Available at https://dx.doi. org/10.21037/apm-21-3228

Data Sharing Statement: Available at https://dx.doi. org/10.21037/apm-21-3228

Conflicts of Interest: Both authors have completed the ICMJE uniform disclosure form (available at https://dx.doi. org/10.21037/apm-21-3228). The authors have no conflicts of interest to declare.

Ethical Statement: The authors are accountable for all aspects of the work in ensuring that questions related to the accuracy or integrity of any part of the work are appropriately investigated and resolved. All procedures performed in this study involving human participants were in accordance with the Declaration of Helsinki (as revised in 2013). The study was approved by the ethics committee of the Institute of Medical Information, Chinese Academy of Medical Sciences \& Peking Union Medical College (the number of the approval: IMICAMS/02/20/HREC) and informed consent was taken from all the participants.

Open Access Statement: This is an Open Access article distributed in accordance with the Creative Commons Attribution-NonCommercial-NoDerivs 4.0 International License (CC BY-NC-ND 4.0), which permits the noncommercial replication and distribution of the article with the strict proviso that no changes or edits are made and the original work is properly cited (including links to both the formal publication through the relevant DOI and the license). See: https://creativecommons.org/licenses/by-nc-nd/4.0/.

\section{References}

1. Yadav KN, Gabler NB, Cooney E, et al. Approximately One In Three US Adults Completes Any Type Of Advance Directive For End-Of-Life Care. Health Aff (Millwood) 2017;36:1244-51.

2. Rietjens JAC, Sudore RL, Connolly M, et al. Definition and recommendations for advance care planning: an international consensus supported by the European Association for Palliative Care. Lancet Oncol 2017;18:e543-51.

3. Cong Y. Rethinking on the Doctor-patient-Relationship. Chinese Medical Ethics 2017;30:665-9.

4. Fan R. Self-determination vs. family-determination: two incommensurable principles of autonomy: a report from East Asia. Bioethics 1997;11:309-22.

5. Fan R, Tao J. Consent to medical treatment: the complex interplay of patients, families, and physicians. J Med Philos 2004;29:139-48.

6. Fan R. Informed consent: why family-oriented? In: Fan R. editor. Family-oriented informed consent: East Asian and American perspectives. Philosophy and Medicine. Cham: Springer International Publishing, 2015:3-23. 
7. Fan R. Confucian reflective equilibrium: Why principlism is misleading for Chinese bioethical decision-making. Asian Bioethics Review 2012;4:4-13.

8. Fan R. Contemporary Confucian bioethics. Beijing: Peking University Press, 2011.

9. Wu J, Fan R. Conscience opposition: A new theory of Confucian ethics for the aged. Studies in Ethics 2017;(2):118-24.

10. Wu F, Wen H, Tao W, et al. Family participation in medical decision-making. China Medical Administration Sciences 2020;10:68-72.

11. Zhao J, Chen G, Xiong Y. Investigation on the Effect of Doctor-patient Joint Decision-making Model in Promoting Mutual Trust Between Elderly Patients and Doctors. Medical Information 2021;34:142-4.

12. Liu X, Gerald H, Yang M, et al. Construction and Implementation Strategies of the "Family-Clinician Shared Decision-Making" Model. Chinese Hospital Management 2021;41:55-9.

13. Huang Y, Wei Z, Cong Y. An Empirical Investigation and Ethical Discussion on the Optimal Decision-making for Cancer Treatment. Medicine \& Philosophy 2017;38:95-7.

14. Gao Q, Hu J, He D, et al. Consistency on End-of-Life Decision-making in Terminal-stage patients and their families. Journal of Nursing Science 2019;34:80-3.

15. Lee JK, Keam B, An AR, et al. Surrogate decision-making in Korean patients with advanced cancer: a longitudinal study. Support Care Cancer 2013;21:183-90.

16. Ivo K, Younsuck K, Ho YY, et al. A survey of the perspectives of patients who are seriously ill regarding end-of-life decisions in some medical institutions of Korea, China and Japan. J Med Ethics 2012;38:310-6.

17. Deng RL, Duan JZ, Zhang JH, et al. Advance care planning for frail older people in China: A discussion paper. Nurs Ethics 2019;26:1696-706.

18. Lambert South A, Elton J. Contradictions and Promise for End-of-Life Communication among Family and Friends: Death over Dinner Conversations. Behav Sci (Basel) 2017;7:24.

19. Qiong Y. Before the snow fell: My last lesson in life. Peking: China Friendship Publishing Company, 2018.

20. Matarasso Greenfeld S, Gil E, Agmon M. A bridge to cross: Tube feeding and the barriers to implementation of palliative care for the advanced dementia patient. J Clin Nurs 2020. [Epub ahead of print].

21. Porteri C. Advance directives as a tool to respect patients' values and preferences: discussion on the case of Alzheimer's disease. BMC Med Ethics 2018;19:9.
22. Vollmann J. Advance directives in patients with Alzheimer's disease. Ethical and clinical considerations. Med Health Care Philos 2001;4:161-7.

23. Willmott L, White B, Mathews B. Law, autonomy and advance directives. J Law Med 2010;18:366-89.

24. Dworkin R. Autonomy and the demented self. Milbank Q 1986;64:4-16.

25. Dresser R. The Limited Value of Dementia-Specific Advance Directives. Hastings Cent Rep 2021;51:4-5.

26. Brody H, Hermer LD, Scott LD, et al. Artificial nutrition and hydration: the evolution of ethics, evidence, and policy. J Gen Intern Med 2011;26:1053-8.

27. Mitchell SL, Teno JM, Roy J, et al. Clinical and organizational factors associated with feeding tube use among nursing home residents with advanced cognitive impairment. JAMA 2003;290:73-80.

28. Sampson EL, Candy B, Jones L. Enteral tube feeding for older people with advanced dementia. Cochrane Database Syst Rev 2009;(2):CD007209.

29. Palecek EJ, Teno JM, Casarett DJ, et al. Comfort feeding only: a proposal to bring clarity to decision-making regarding difficulty with eating for persons with advanced dementia. J Am Geriatr Soc 2010;58:580-4.

30. Mitchell SL, Mor V, Gozalo PL, et al. Tube Feeding in US Nursing Home Residents With Advanced Dementia, 2000-2014. JAMA 2016;316:769-70.

31. Yan Y, Zhang H, Gao W, et al. Current awareness of palliative care in China. Lancet Glob Health 2020;8:e333-5.

32. Jaafar MH, Mahadeva S, Tan KM, et al. Long-Term Nasogastric Versus Percutaneous Endoscopic Gastrostomy Tube Feeding in Older Asians With Dysphagia: A Pragmatic Study. Nutr Clin Pract 2019;34:280-9.

33. Gomes CA Jr, Andriolo RB, Bennett C, et al. Percutaneous endoscopic gastrostomy versus nasogastric tube feeding for adults with swallowing disturbances. Cochrane Database Syst Rev 2015;(5):CD008096.

34. Dwolatzky T, Berezovski S, Friedmann R, et al. A prospective comparison of the use of nasogastric and percutaneous endoscopic gastrostomy tubes for long-term enteral feeding in older people. Clin Nutr 2001;20:535-40.

35. Ad-Hoc PEG Tube Study Group. When to Recommend a PEG Tube: A Decision Tree for Clinicians from a Catholic Perspective. Linacre Q 2012;79:25-40.

36. Cuadro CKA, Herrera JAO, Quiros TL. To be alive vs. to live: Filipino elderly, nasogastric tubes, and the issue of artificial feeding in dementia: Long-term care and ethics. Alzheimers Dement 2020;16:e044200. 
37. Lin LC, Li MH, Watson R. A survey of the reasons patients do not chose percutaneous endoscopic gastrostomy/jejunostomy (PEG/PEJ) as a route for longterm feeding. J Clin Nurs 2011;20:802-10.

38. Murray S. The 2015 Quality of Death Index: Ranking palliative care across the world-A report by The Economist Intelligence Unit. London: The Economist, 2015.

39. van der Smissen D, Overbeek A, van Dulmen S, et al. The Feasibility and Effectiveness of Web-Based Advance Care Planning Programs: Scoping Review. J Med Internet Res

Cite this article as: Huang Y, Liu H. Is there a need for advance care planning in China? - an interview survey of healthcare professionals in the neurology department. Ann Palliat Med 2021;10(11):11918-11930. doi: 10.21037/apm-21-3228 2020;22:e15578.

40. Rolnick JA, Asch DA, Halpern SD. Delegalizing Advance Directives - Facilitating Advance Care Planning. N Engl J Med 2017;376:2105-7.

41. Kim H, Im HS, Lee KO, et al. Changes in decisionmaking process for life-sustaining treatment in patients with advanced cancer after the life-sustaining treatment decisions-making act. BMC Palliat Care 2021;20:63.

(English Language Editor: B. Draper) 